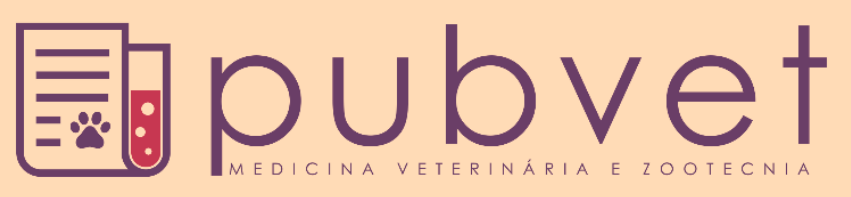

https://doi.org/10.31533/pubvet.v13n12a480.1-13

\title{
Suplementação com virginiamicina e monensina em dietas de vacas leiteiras com alta inclusão de concentrado
}

\author{
Juliana Santos Silva ${ }^{1 * \bullet}$, Victor Marco Rocha Malacco ${ }^{1}$ (D), Thiago Campos Escarce $^{1 \bullet}$, Rafael

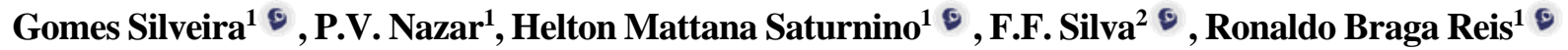 \\ ${ }^{1}$ Departamento de Zootecnia, Universidade Federal de Minas Gerais, Minas Gerais, 30161-970, Brasil \\ ${ }^{2}$ Departamento de Zootecnia, Universidade Federal de Viçosa, Minas Gerais, 36570-900, Brasil \\ *Autor correspondente: juliana.vet88@gmail.com
}

Resumo. Objetivou-se avaliar o desempenho, as digestibilidades aparentes dos nutrientes e os parâmetros de fermentação ruminal em dietas de vacas leiteiras com alta inclusão de concentrado suplementadas com virginiamicina e/ou monensina. Foram utilizadas cinco vacas mestiças fistuladas no rúmem, com 88 dias de lactação, produção média diária de leite de $26 \mathrm{~kg}$ peso corporal médio de $550 \mathrm{~kg}$ no início do experimento em delineamento de quadrado latino $5 \times 5$. As dietas experimentais diferiam quanto à inclusão e proporção destes aditivos: $300 \mathrm{mg} / \mathrm{d}$ de $\mathrm{VM}\left(\mathrm{VM}^{300}\right) ; 300 \mathrm{mg} / \mathrm{d}$ de $\mathrm{MO}\left(\mathrm{MO}^{300}\right) ; 300 \mathrm{mg} / \mathrm{d}$ de $\mathrm{VM}$ e $300 \mathrm{mg} / \mathrm{d}$ de $\mathrm{MO}\left(\mathrm{VM}^{300}+\mathrm{MO}^{300}\right) ; 300 \mathrm{mg}$ de $\mathrm{VM}$ e $150 \mathrm{mg}$ de $\mathrm{MO}\left(\mathrm{VM}^{300}+\mathrm{MO}^{150}\right)$; Controle, sem aditivos (C). Não foi observada diferença no consumo de MS e nutrientes, com exceção para o consumo de amido que foi maior no grupo controle quando comparado com o grupo VM e também foi observada uma tendência para maior consumo de amido no grupo $\mathrm{VM}^{300}+\mathrm{MO}^{300}$ quando comparado ao grupo $\mathrm{VM}^{300}+\mathrm{MO}^{150}$. Houve diferença para a digestibilidade aparente da proteína bruta, indicando superioridade dos grupos que utilizaram a associação de VM e MO quando comparados ao grupo suplementado apenas com VM. Foi observada maior produção de leite ao grupo controle e aos grupos suplementados com VM e MO associadas quando comparados a VM. Os dados de eficiência alimentar evidenciaram melhores valores para os grupos controle e os que utilizaram os aditivos associados. Valores de NUP e nitrogênio amoniacal foram superiores nas dietas que utilizaram VM e MO associadas. Houve superioridade dos animais suplementados com a VM e a MO associadas nas doses de 300 e $150 \mathrm{mg} / \mathrm{vaca} / \mathrm{d}$, respectivamente, em manter os valores de $\mathrm{pH}$ ruminal mais elevados quando comparado a associação na dose de $300 \mathrm{mg} / \mathrm{vaca} / \mathrm{dia}$ de VM e MO. O mesmo comportamento foi observado para $\mathrm{pH}$ fecal. Foram observadas maiores produções de propionato, butirato e AGV totais no grupo controle quando comparado ao grupo VM.

Palavras chave: Aditivos, produção leite, eficiência alimentar, fermentação, rúmen

\section{Virginiamycin and monensin supplementation in high-concentrate dairy cow's diets}

Abstract. The objective of this study was to evaluate the performance, apparent digestibility of nutrients and ruminal fermentation parameters in diets of high concentration for dairy cows supplemented with virginiamycin or monensin. Five rumen-fistulated crossbred cows with 88 days of lactation, average daily milk yield of $26 \mathrm{~kg}$, mean body weight of $550 \mathrm{~kg}$ at the beginning of the experiment in a $5 \times 5$ Latin square design, were used. Proportion of these additives: $300 \mathrm{mg} / \mathrm{d}$ VM (VM300); $300 \mathrm{mg} / \mathrm{d}$ MO (MO300); 300 $\mathrm{mg} / \mathrm{d} \mathrm{MV}$ and $300 \mathrm{mg} / \mathrm{d} \mathrm{OM}$ (VM300 + MO300); $300 \mathrm{mg}$ of $\mathrm{VM}$ and $150 \mathrm{mg}$ of MO (VM300 + MO150); Control, without additives (C). There was no difference in DM and 
nutrient intake, except for starch intake which was higher in the control group when compared to the VM group and a trend towards higher starch intake in the VM300 + MO300 group as compared to the group VM300 + MO150. There was a difference in the apparent digestibility of crude protein, indicating superiority of the groups that used the association of MV and OM when compared to the group supplemented only with MV. Higher milk yield was observed in the control group and in the groups supplemented with MV and associated OM when compared to MV. Food efficiency data showed better values for the control groups and those that used the associated additives. NUP and ammonia nitrogen values were higher in diets that used associated MV and OM. There was a superiority of animals supplemented with MV and associated OM at doses of 300 and 150 $\mathrm{mg} / \mathrm{cow} / \mathrm{d}$, respectively, in maintaining higher ruminal $\mathrm{pH}$ values when compared to the combination at $300 \mathrm{mg} / \mathrm{cow} / \mathrm{day}$ of MV and MO. The similar behavior was observed for fecal $\mathrm{pH}$. Higher total propionate, butyrate and AGV productions were observed in the control group when compared to the VM group.

Keywords: Additives, milk production, food efficiency, fermentation, rumen

\section{Suplementos de virginiamicina y monensina en dietas de vacas lecheras con alto contenido de concentrado}

Resumen. El objetivo de este estudio fue evaluar el rendimiento, la digestibilidad aparente de los nutrientes y los parámetros de fermentación ruminal en las dietas de vacas lecheras con alta concentración suplementadas con virginiamicina y/o monensina. Se utilizaron cinco vacas mestizas fistuladas en el rumen con 88 días de lactancia, rendimiento promedio diario de leche de $26 \mathrm{~kg}$, peso corporal promedio de $550 \mathrm{~kg}$ al comienzo del experimento en un diseño cuadrado latino de 5 x 5 . Las dietas experimentales diferían en su inclusión y proporción de estos aditivos: $300 \mathrm{mg} / \mathrm{d} \mathrm{VM}\left(\mathrm{VM}^{300}\right) ; 300 \mathrm{mg} / \mathrm{d} \mathrm{MO}\left(\mathrm{MO}^{300}\right) ; 300 \mathrm{mg} / \mathrm{d}$ $\mathrm{VM}$ y $300 \mathrm{mg} / \mathrm{d} \mathrm{MO}\left(\mathrm{VM}^{300}+\mathrm{MO}^{300}\right) ; 300 \mathrm{mg}$ de $\mathrm{VM}$ y $150 \mathrm{mg}$ de $\mathrm{MO}\left(\mathrm{VM}^{300}+\mathrm{MO}^{150}\right)$; Control, sin aditivos (C). No hubo diferencias en la ingesta de materia seca y nutrientes, excepto en la ingesta de almidón, que fue mayor en el grupo control en comparación con el grupo VM y una tendencia hacia una mayor ingesta de almidón en el grupo VM300 + MO300 en comparación con el grupo. VM300 + MO150. Los valores aparentes de digestibilidad de la proteína cruda fueron diferentes, indicando superioridad de los grupos que usaron la asociación de VM y MO en comparación con el grupo suplementado solo con VM. Se observó una mayor producción de leche en el grupo control y en los grupos suplementados con VM y MO asociada en comparación con VM. Los datos de eficiencia alimentaria mostraron mejores valores para los grupos de control y aquellos que usaron los aditivos asociados. Los valores de NUP y de nitrógeno amoniacal fueron mayores en las dietas que usaron VM y MO asociados. Hubo una superioridad de los animales suplementados con VM y MO asociada a dosis de 300 y $150 \mathrm{mg} /$ vaca / día, respectivamente, en el mantenimiento de valores de $\mathrm{pH}$ ruminal más altos en comparación con la combinación de $300 \mathrm{mg} /$ vaca / día de VM. y MO. Se observó el mismo comportamiento para el $\mathrm{pH}$ fecal. Se observaron mayores producciones totales de propionato, butirato y AGV en el grupo de control en comparación con el grupo VM.

Palabras clave: aditivos, producción de leche, eficiencia alimentaria, fermentación rumen

\section{Introdução}

A produção mundial de leite cresce desde o ano de 2009. Segundo dados do Departamento de Agricultura dos Estados Unidos (USDA), o volume global atingiu 596,31 bilhões de litros. O incremento na oferta mundial foi de $0,7 \%$ em 2016, frente a 2015 e a 1,8\% em 2017 na comparação com 2016. Processo este que vem acompanhado do aumento da produtividade das vacas leiteiras e consequentemente aumento da inclusão de concentrados na dieta, além da busca por uma melhor eficiência alimentar. Contudo, as dietas ricas em carboidratos não fibrosos, de fermentação rápida, particularmente as que contêm quantidades elevadas de grãos, via de regra levam a quedas significativas 
no pH ruminal (González et al., 2012; Wu \& Papas, 1997). Nesse contexto, torna-se prioritário, após conhecer os requisitos nutricionais dos animais e o valor nutritivo dos alimentos que farão parte de sua alimentação, a busca por melhoria na eficiência de utilização das dietas por esses animais, para que os alimentos sejam transformados de maneira mais eficiente e econômica em produtos que venham atender às demandas da população humana. Assim, manipular o processo de fermentação ruminal é uma maneira particular de alterar o metabolismo normal dos ruminantes, visando o aperfeiçoamento do desempenho dos animais. Desta forma, os aditivos alimentares são importantes, pois podem causar inúmeros benefícios ao sistema de produção, como ganho de peso, melhora na conversão alimentar, diminuição do risco de acidose, melhora a resposta imunológica, o que resulta em ganhos para o sistema de produção (Callaway et al., 2003; McGuffey et al., 2001).

Neste contexto, destaca se a virginiamicina, que em comparação aos outros compostos como, salonimicina e monensina, tem se mostrado mais eficaz em reduzir a produção de ácido lático uma vez que possui ação direta sobre as espécies produtoras deste composto (Nagaraja et al., 1997; Nagaraja \& Taylor, 1987).

Por outro lado, a monensina age sobre bactérias gram-positivo, influenciando a fermentação ruminal e gerando alterações na proporção de ácidos graxos voláteis, favorecendo a produção de propionato. A mudança na proporção de ácidos graxos voláteis também resulta em uma diminuição na produção de gás metano, tornando mais eficiente o metabolismo energético do animal (Duffield et al., 2012; Schelling, 1984).

No entanto, pouco se sabe sobre a interação de ambas as moléculas sobre os efeitos nos padrões de fermentação e desempenho de vacas leiteiras. Portanto, objetivou-se avaliar o desempenho, as digestibilidades aparentes dos nutrientes e os parâmetros de fermentação ruminal em dietas de vacas leiteiras com alta inclusão de concentrado suplementadas com virginiamicina e/ou monensina.

\section{Material e métodos}

\section{Local e época do experimento:}

O experimento foi conduzido no período de 03 de setembro a 16 de fevereiro de 2016, na fazenda experimental de Universidade Federal de Minas Gerais (UFMG). A média anual de temperatura é de $20,0^{\circ}$ C. Todos os procedimentos realizados seguiram as orientações do comitê de ética na experimentação animal da UFMG.

\section{Animais, instalações e delineamento experimental:}

Foram utilizadas cinco vacas mestiças (Holandês x Gir) fistuladas no rúmem, com $88 \pm 26$ dias de lactação, produção média diária de leite de $26 \pm 6,0 \mathrm{~kg}$ peso corporal médio de $550 \pm 58 \mathrm{~kg}$ no início do experimento. $\mathrm{O}$ delineamento utilizado foi o quadrado latino $5 \times 5$, cinco períodos experimentais de 28 dias cada, sendo 21 dias de adaptação às dietas experimentais e sete dias de coletas de dados e amostras. Os animais foram confinados em instalações tipo Tie Stall e distribuídos aleatoriamente entre as dietas experimentais. A estrutura de cocho possuía 10 divisórias, as vacas foram distribuídas saltando uma cama e com uma divisória no cocho que impedia o acesso a dieta dos animais vizinhos. Os animais tinham acesso livre ao alimento e a água em bebedouros automatizados e individualizados.

\section{Dietas experimentais e manejo dos animais:}

As dietas experimentais foram balanceadas, segundo o NRC (2001) para serem isoprotéicas e isoenergéticas, e encontram-se na tabela 1. Os aditivos virginiamicina (VM) (Eskalin®, Phibro Animal Health Corporation, SP, BR) e monensina sódica (MO) (Bovensin ${ }^{\circledR}$, Phibro Animal Health Corporation, $\mathrm{SP}, \mathrm{BR}$ ) foram incluídos, junto ao núcleo vitamínico mineral. As dietas experimentais diferiam quanto à inclusão e proporção dos aditivos: $300 \mathrm{mg} / \mathrm{d}$ de $\mathrm{VM}\left(\mathrm{VM}^{300}\right) ; 300 \mathrm{mg} / \mathrm{d}$ de $\mathrm{MO}\left(\mathrm{MO}^{300}\right) ; 300 \mathrm{mg} / \mathrm{d}$ de $\mathrm{VM}$ e $300 \mathrm{mg} / \mathrm{d}$ de $\mathrm{MO}\left(\mathrm{VM}^{300}+\mathrm{MO}^{300}\right) ; 300 \mathrm{mg}$ de $\mathrm{VM} \mathrm{e} 150 \mathrm{mg}$ de $\mathrm{MO}\left(\mathrm{VM}^{300}+\mathrm{MO}^{150}\right)$ e Controle, sem aditivos (CON).

As dietas foram oferecidas duas vezes ao dia, às 06:30 e 16:30 horas, logo que as vacas retornavam das ordenhas. A quantidade era ajustada diariamente para obter 5\% de sobra. 
As vacas receberam $500 \mathrm{mg}$ de somatotropina bovina recombinante (BSTr) (BOOSTIN ${ }^{\circledR} 500 \mathrm{mg}-$ MSD Saúde Animal, SP, BR) a cada 14 dias, na fossa ísquio retal, alternando-se os lados a cada aplicação.

Tabela 1. Formulação e composição química das dietas experimentais de vacas leiteiras com ou sem inclusão de virginiamicina e/ou monensina

\begin{tabular}{|c|c|c|c|c|c|}
\hline \multirow{3}{*}{ Ingredientes } & \multicolumn{5}{|l|}{ Dietas $^{1}$} \\
\hline & $\mathrm{VM}$ & $\mathrm{MO}$ & $\mathrm{VM}^{300}+\mathrm{MO}^{300}$ & $\mathrm{VM}^{300}+\mathrm{MO}^{150}$ & $\mathrm{C}$ \\
\hline & \multicolumn{5}{|l|}{ Concentração, \% MS } \\
\hline Cana de açúcar & 32,98 & 32,98 & 32,98 & 32,98 & 32,98 \\
\hline Milho, moído & 17,82 & 17,82 & 17,82 & 17,82 & 17,82 \\
\hline Soja, farelo & 22,26 & 22,26 & 22,26 & 22,26 & 22,26 \\
\hline Polpa & 17,02 & 17,02 & 17,02 & 17,02 & 17,02 \\
\hline Algodão, caroço & 10,80 & 10,80 & 10,80 & 10,80 & 10,80 \\
\hline Ureia & 0,34 & 0,34 & 0,34 & 0,34 & 0,34 \\
\hline Premix $^{2}$ & 2,83 & 2,83 & 2,83 & 2,83 & 2,83 \\
\hline Eskalin $®$ & 0,075 & - & 0,075 & 0,075 & - \\
\hline Bovensin ${ }^{\circledR}$ & - & 0,075 & 0,075 & 0,0038 & - \\
\hline \multicolumn{6}{|l|}{ Nutrientes ${ }^{3}, \% \mathrm{MS}$} \\
\hline$\overline{\mathrm{PB}}$ & 16 & 16 & 16 & 16 & 16 \\
\hline PDR & 10,6 & 10,6 & 10,6 & 10,6 & 10,6 \\
\hline PNDR & 5,4 & 5,4 & 5,4 & 5,4 & 5,4 \\
\hline FDN & 28,7 & 28,7 & 28,7 & 28,7 & 28,7 \\
\hline FDNf & 16,2 & 16,2 & 16,2 & 16,2 & 16,2 \\
\hline FDA & 20,3 & 20,3 & 20,3 & 20,3 & 20,3 \\
\hline $\mathrm{EL}(\mathrm{Mcal} / \mathrm{kg})$ & 1,47 & 1,47 & 1,47 & 1,47 & 1,47 \\
\hline $\operatorname{BEL}(\mathrm{Mcal} / \mathrm{d})$ & 2,4 & 2,4 & 2,4 & 2,4 & 2,4 \\
\hline NDT & 71 & 71 & 71 & 71 & 71 \\
\hline $\mathrm{CNF}$ & 44,8 & 44,8 & 44,8 & 44,8 & 44,8 \\
\hline Amido & 15 & 15 & 15 & 15 & 15 \\
\hline $\mathrm{EE}$ & 3,9 & 3,9 & 3,9 & 3,9 & 3,9 \\
\hline \multicolumn{6}{|c|}{ Balanço proteico, g/d (Proteína Metabolizável) } \\
\hline Exigência & 2068 & 2068 & 2068 & 2068 & 2068 \\
\hline Suprido & 2068 & 2068 & 2068 & 2068 & 2068 \\
\hline Balanço & 0 & 0 & 0 & 0 & 0 \\
\hline \multicolumn{6}{|c|}{$\mathrm{PDR}$ e $\mathrm{PNDR}^{4}, \mathrm{~g} / \mathrm{d}$} \\
\hline PDR suprida & 2155 & 2155 & 2155 & 2155 & 2155 \\
\hline BPDR & 140 & 140 & 140 & 140 & 140 \\
\hline PNDR suprida & 1019 & 1019 & 1019 & 1019 & 1019 \\
\hline B PNDR & 0 & 0 & 0 & 0 & 0 \\
\hline \multicolumn{6}{|c|}{ 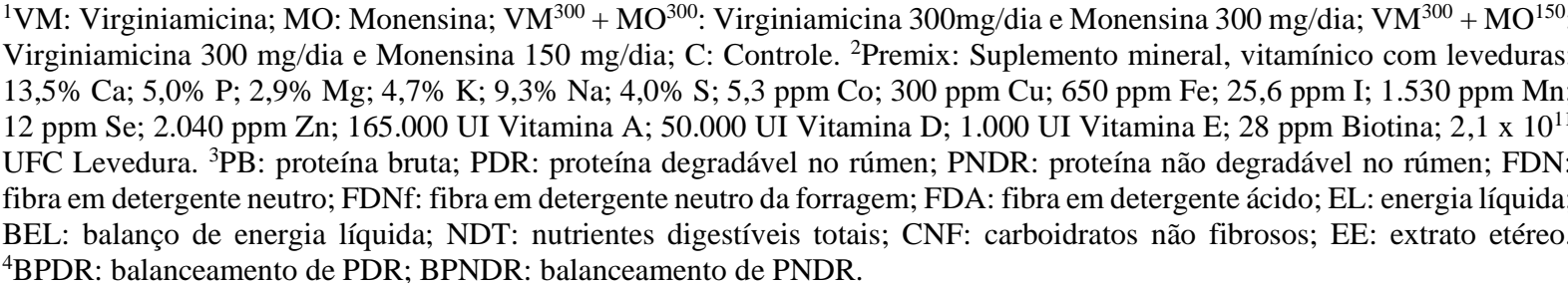 } \\
\hline
\end{tabular}

\section{Determinação do consumo e da digestibilidade aparente dos nutrientes:}

O consumo diário foi determinado, pela diferença entre o peso das dietas oferecidas e das sobras do $22^{\circ}$ ao $28^{\circ}$ dia de cada período experimental.

$\mathrm{O}$ coeficiente de digestibilidade aparente dos nutrientes foi determinado com o uso do método de coleta total das fezes. As coletas foram realizadas durante três períodos de 8 horas a partir do $22^{\circ}$ dia de cada período experimental. As fezes foram coletadas e pesadas concomitantemente à defecação. $\mathrm{O}$ 
segundo e terceiro períodos de amostragem foram atrasados em 8 horas, representando uma coleta de 24 horas. As amostras de fezes foram acondicionadas em sacos plásticos e armazenadas a $-10^{\circ} \mathrm{C}$.

Amostras fecais, das dietas e das sobras foram pré-secas em estufa de ventilação forçada, regulada a $55^{\circ} \mathrm{C}$, por $72 \mathrm{~h}$. As amostras pré-secas foram moídas em moinho estacionário tipo "Thomas Willey" (modelo 4, Arthur H. Thomas Co., Philadelphia PA), montado com peneiras com furos de $1 \mathrm{~mm}$ de diâmetro, e armazenadas em recipientes plásticos para posteriores análises bromatológicas. Quantidades iguais de fezes de cada uma das amostras foram homogeneizadas para confecção de uma amostra composta por vaca por período.

Uma sub-amostra foi levada à estufa a $105^{\circ} \mathrm{C}$ por quatro horas para determinação da matéria seca (MS), de acordo com a AOAC (2005) (método 925.40). O teor de cinzas foi determinado pela queima total de matéria orgânica em mufla a $600^{\circ} \mathrm{C}$ por quatro horas (AOAC, 2005) (método 942.05). O teor de matéria orgânica (MO) foi calculado pela diferença entre a MS e o conteúdo de cinzas. A proteína bruta (PB) foi analisada pelo método de Kjedhall (AOAC International, 2012, método 955.04). O extrato etéreo (EE) foi obtido de acordo com o (AOAC, 2005) (método 920.39). A análise de fibra foi realizada de acordo com o método proposto por Van Soest et al. (1991), para fibra em detergente neutro (FDN) e em detergente ácido (FDA). Os resíduos das análises de FDN e FDA foram submetidos à determinação de cinzas e PB para obtenção dos valores de proteína insolúvel em detergente neutro (PIDN) e proteína insolúvel em detergente ácido (PIDA). Esses valores foram usados para corrigir a FDN e a FDA para cinzas e proteína. O percentual de carboidratos não fibrosos (CNF) foi calculado pela equação proposta pelo NRC (2001): $\mathrm{CNF}=100-\left(\left(\% \mathrm{FDN}-\% \mathrm{FDN}_{\mathrm{PB}}\right)+\% \mathrm{~PB}+\% \mathrm{EE}+\%\right.$ Cinzas $)$. A determinação do amido foi efetuada pelo método enzimático, segundo metodologia descrita por Hall et al. (1999). A concentração de proteína degradável no rúmen (PDR), proteína não degradável no rúmen (PNDR), energia líquida (EL), proteína metabolizável (PM) foram estimadas utilizando o modelo do NRC (2001) com base no consumo de matéria seca (CMS) observada, a produção e a composição do leite e o peso de vaca.

\section{Avaliação do desempenho}

Os animais foram ordenhados duas vezes ao dia, às 07:00 e às 16:00 h, em sistema de ordenha mecânica com contenção do tipo espinha de peixe, duplo quatro, modelo Dematron ${ }^{\circledR}$ 70/75 (GEA Farm Technologies Inc.). A produção de leite foi mensurada em medidores (GEA GEA Farm Technologies Inc.), considerando-se o leite produzido em 14 ordenhas consecutivas, do $22^{\circ}$ ao $28^{\circ}$ dia de cada período experimental. Amostras de leite, das seis últimas ordenhas, foram coletadas com auxílio de coletor acoplado a linha do leite, acondicionadas em frascos plásticos com 2-bromo 2-nitropropano 1,3-diol na relação de $10 \mathrm{mg}$ para $50 \mathrm{~mL}$ de leite, mantidas sob refrigeração $\left(4^{\circ} \mathrm{C}\right)$ e enviadas para análise no Laboratório de Qualidade do Leite da Universidade Federal de Minas Gerais. As amostras de leite foram analisadas quanto aos teores de gordura, proteína e lactose pelo método de infravermelho (Bentley 2000 Bentley Instruments Inc., Chaska, MN) (IDF, 2000) e nitrogênio ureico do leite (NUL) pela metodologia Infrared de Fourier Transform (CombiScope FTIR advanced/Delta Instruments, Drachten, Netherlands).

O leite foi corrigido para o teor de 3,5\% de gordura, conforme a fórmula proposta pelo NRC (2001): $\mathrm{PLC}_{3,5 \%}=(0,35 \times$ Produção de Leite $)+(15 \times(\%$ Gordura/100) $\mathrm{x}$ Produção de Leite $)$. A secreção diária de energia no leite foi calculada pela equação (NRC, 2001): $[(0,0929 \times \%$ gordura $)+(0,0547 \times \%$ de proteína $)+(0,0395 \times$ \% de lactose $)] \times$ kg de leite. A produção de leite corrigida para energia (LCE) foi calculada pela equação: Leite $\mathrm{E}=$ EnLeite/0,70, assumindo que o conteúdo de energia em leite com $3,7 \%$ de gordura, $3,2 \%$ de proteína e $4,6 \%$ de lactose e $0,70 \mathrm{Mcal} / \mathrm{kg}$.

Os cálculos da eficiência alimentar e da eficiência da utilização do $\mathrm{N}$ foram realizados segundo as equações propostas por DePeters \& Ferguson (1992).

\section{Determinação dos parâmetros ruminais e pH fecal}

Para a determinação da concentração de amônia $\left(\mathrm{N}^{\left.-\mathrm{NH}_{3}\right)}\right)$, do $\mathrm{pH}$ e das concentrações de ácidos graxos voláteis (AGV) as amostras de fluído ruminal foram coletadas manualmente, diretamente do rúmen pela fístula ruminal e filtradas em gaze, no $25^{\circ}$ dia de cada período experimental. Os tempos de coleta foram imediatamente antes da alimentação $(0)$ e a cada $2 \mathrm{~h}$, totalizando 24 horas de coleta. 
Foi realizada leitura imediata do $\mathrm{pH}$ do líquido ruminal utilizando-se um medidor de $\mathrm{pH}$ Piccolo 2 (Hanna Instruments, Woonsocket, RI), frequentemente ajustado para o padrão $\mathrm{pH}$ de 4,0 e 7,0 a cada leitura. Logo após, $8 \mathrm{~mL}$ do líquido filtrado foi acondicionado em frasco âmbar que continham $2 \mathrm{~mL}$ de ácido metafosfórico 25\% para mensuração dos AGV. Outra alíquota de $50 \mathrm{ml}$ foi armazenada com 1 $\mathrm{mL}$ de ácido sulfúrico (50\%) para dosagem do teor de $\mathrm{N}_{-} \mathrm{NH}_{3}(\mathrm{mg} / \mathrm{dL}$ ) (Detmann et al., 2012). As amostras coletadas foram armazenadas a $-10^{\circ} \mathrm{C}$ para posteriores análises. Ao final do ensaio, as amostras foram descongeladas à temperatura ambiente, e concentração de $\mathrm{N}-\mathrm{NH}_{3}$ medida após a centrifugação do líquido ruminal, utilizando destilação com óxido de magnésio, e usando ácido bórico como solução receptora e ácido clorídrico 0,01 N na titulação (Detmann et al., 2012).

A análise de AGV foi realizada por cromatografia gasosa em aparelho Shimadzu GC-17 $A^{\circledR}$ (Shimadzu do Brasil, SP, BR).

$\mathrm{O} \mathrm{pH}$ fecal foi determinado no $24^{\circ}$ e $25^{\circ}$ dia de cada período experimental, utilizando medidor de $\mathrm{pH}$ Piccolo 2 (Hanna Instruments, Woonsocket, RI). Foram coletadas amostras fecais de cada vaca, e uma subamostra de $20 \mathrm{~g}$ foi colocada em um frasco plástico, adicionado $20 \mathrm{~mL}$ de água destilada deionizada e realizada filtração em gaze para posterior mensuração do $\mathrm{pH}$.

Para mensuração dos teores de nitrogênio ureico no plasma (NUP) amostras de sangue foram coletadas nos vasos coccígeos no último dia de cada período experimental ( $28^{\circ}$ dia $)$, utilizando tubo evacuado (Vacutainer ${ }^{\circledR}$, Becton Dickinson, BR) com EDTA 0, 2, 4, 6, 8, 12 e 16h após a alimentação. O sangue foi centrifugado a $3000 \mathrm{x}$ g por 15 minutos e o plasma foi congelado em microtubos Eppendorf (Bioplast $^{\circledR}$, Rio de Janeiro, RJ, Brasil) para posterior análise.

Os teores de ureia foram determinados por método colorimétrico enzimático UV (GLDH) com kits específicos (Ureia UV reagente R1, Kovalent do Brasil Ltda., Rio de Janeiro, BR). As leituras foram realizadas usando o analisador clínico Cobas Mira (Global Medical Instrumentations, Inc., Ramsey, MN, EUA).

\section{Análise estatística:}

As análises estatísticas foram realizadas segundo o PROC MIXED do programa estatístico SAS (2004) (Institute Inc., Cary, NC, versão 9.4), pelos modelos matemáticos 1 e 2.

\section{Modelo 1.}

Ajustado aos dados de todas as variáveis que não apresentam medidas repetidas no tempo, como: dados de desempenho e digestibilidade aparente dos nutrientes:

$\mathrm{Y}_{\mathrm{ijk} \mathrm{l}}=\mu+\mathrm{P}_{\mathrm{j}}+\mathrm{V}_{\mathrm{k}(\mathrm{i})}+\mathrm{T}_{1}+\mathrm{PT}_{\mathrm{j} \mathrm{l}}+\mathrm{E}_{\mathrm{ijk} \mathrm{l}}$

Onde: $\mathrm{Y}_{\mathrm{ijkl}}=$ variável dependente; $\mu=$ média geral; $\mathrm{P}_{\mathrm{j}}=$ efeito do período $\mathrm{j} ; \mathrm{V}_{\mathrm{k}(\mathrm{i})}=$ efeito do animal $\mathrm{k}$ (dentro do quadrado); $\mathrm{T}_{1}=$ efeito do tratamento $1 ; \mathrm{PT}_{\mathrm{jl}}=$ interação entre período $\mathrm{j}$ e tratamento $\mathrm{l} ; \mathrm{E}_{\mathrm{ijkl}}=$ erro associado a cada observação, assumindo uma distribuição normal e independente dos dados.

\section{Modelo 2.}

Ajustado aos dados de todas as variáveis que apresentam medidas repetidas no tempo, como: parâmetros sanguíneos e ruminais.

$$
\mathrm{Y}_{\mathrm{ijklm}}=\mu+\mathrm{P}_{\mathrm{j}}+\mathrm{V}_{\mathrm{k}(\mathrm{i})}+\mathrm{T}_{1}+\mathrm{PT}_{\mathrm{jl}}+\mathrm{E}_{\mathrm{ijkl}}+\mathrm{Z}_{\mathrm{m}}+\mathrm{ZT}_{\mathrm{ml}}+\mathrm{E}_{\mathrm{ijklm}},
$$

Onde: $\mathrm{Y}_{\mathrm{ijklm}}=$ variável dependente; $\mu=$ média geral; $\mathrm{P}_{\mathrm{j}}=$ efeito do período $\mathrm{j} ; \mathrm{V}_{\mathrm{k}(\mathrm{i})}=$ efeito do animal $\mathrm{k}$; $\mathrm{T}_{1}=$ efeito do tratamento $1 ; \mathrm{PT}_{\mathrm{jl}}=$ interação entre período $\mathrm{j}$ e tratamento $1 ; \mathrm{E}_{\mathrm{j} \mathrm{jk}}=$ erro associado a cada observação, assumindo uma distribuição normal e independente dos dados; $Z_{\mathrm{m}}=$ efeito do tempo $\mathrm{m}$; $\mathrm{ZT}_{\mathrm{ml}}=$ interação entre tempo $\mathrm{m}$ e tratamento $1 ; \mathrm{E}_{\mathrm{ijk} \mathrm{klm}}=$ erro associado a cada sub parcela, assumindo uma distribuição normal e independente dos dados.

A estrutura de covariância AR (1) de medidas repetidas foi escolhida de acordo com os Critérios de Informação de Akaike (AICc) mais baixos corrigidos. Em todos os modelos, os efeitos dos níveis de YDP foram testados com linear polinomial $(-3,-1,1,3)$, quadrático $(1,-1,-1,1)$ e cúbico $(-1,+3,-3$, $+1)$ contrastes. 
As médias foram comparadas por contrastes ortogonais e a significância dos efeitos foi considerada em $\mathrm{P}<0,05$ e os valores de $\mathrm{P}$ de 0,05 a 0,10 foram interpretados como tendência. Os contrastes utilizados foram: contraste $1(\mathrm{C} 1)$ : VM x MO; contraste $2(\mathrm{C} 2)$ : VM x C; contraste $3(\mathrm{C} 3)$ : $\mathrm{VM} \times \mathrm{VM}^{300}+\mathrm{MO}^{300} \mathrm{e}$ $\mathrm{VM}^{300}+\mathrm{MO}^{150}$ e contraste $4(\mathrm{C} 4): \mathrm{VM}^{300}+\mathrm{MO}^{300} \times \mathrm{VM}^{300}+\mathrm{MO}^{150}$.

\section{Resultados e discussão}

\section{Consumo e digestibilidade aparente dos nutrientes:}

As dietas experimentais foram formuladas para atender as exigências do NRC (2001) e serem isoprotéicas (16\% de PB) e isoenergéticas (71\% de NDT). Não foi observada diferença no consumo de nutrientes para as dietas experimentais $(\mathrm{P}>0,05$; Tabela 2$)$, com exceção para o consumo de amido que foi maior no grupo controle quando comparado com o grupo VM e também foi observada uma tendência para maior consumo de amido no grupo $\mathrm{VM}^{300}+\mathrm{MO}^{300}$ quando comparado ao grupo $\mathrm{VM}^{300}+\mathrm{MO}^{150}$, o que justifica também o maior consumo de NDT encontrado para este grupo. Esta diferença de maior consumo de amido pelo grupo controle pode ser explicada por uma possível ingestão seletiva destes animais que consumiam o concentrado em detrimento ao volumoso.

Tabela 2. Consumo e digestibilidade aparente dos nutrientes em dietas de vacas leiteiras com ou sem inclusão de virginiamicina e/ou monensina

\begin{tabular}{|c|c|c|c|c|c|c|c|c|c|c|}
\hline \multirow{2}{*}{ Itens $^{1}$} & \multicolumn{5}{|c|}{ Dietas $^{2}$} & \multirow{2}{*}{$\mathrm{EPM}^{3}$} & \multicolumn{4}{|c|}{ Contrastes $^{4}($ Valor de $\mathrm{P})$} \\
\hline & VM & MO & $\mathrm{VM}^{300}+\mathrm{MO}^{300}$ & $\mathrm{VM}^{300}+\mathrm{MO}^{150}$ & $\mathrm{C}$ & & $\mathrm{C} 1$ & $\mathrm{C} 2$ & $\mathrm{C} 3$ & $\mathrm{C} 4$ \\
\hline \multicolumn{11}{|c|}{ Consumo dos Nutrientes, $\mathrm{kg}$ de MS/d } \\
\hline $\mathrm{CMS}, \mathrm{kg} / \mathrm{d}$ & 16,25 & 16,35 & 17,06 & 16,38 & 17,06 & 0,35 & 0,78 & 0,12 & 0,29 & 0,19 \\
\hline CMS, \% PV & 3,45 & 3,42 & 3,56 & 3,45 & 3,53 & 0,29 & 0,67 & 0,19 & 0,24 & 0,08 \\
\hline $\mathrm{CMO}, \mathrm{kg} / \mathrm{d}$ & 17,20 & 17,08 & 17,42 & 17,42 & 17,51 & 0,19 & 0,65 & 0,27 & 0,36 & 1,00 \\
\hline $\mathrm{CPB}$ & 2,61 & 2,66 & 2,69 & 2,59 & 2,65 & 0,05 & 0,53 & 0,54 & 0,60 & 0,16 \\
\hline CFDN & 6,19 & 6,14 & 6,55 & 6,15 & 6,55 & 0,02 & 0,83 & 0,21 & 0,51 & 0,16 \\
\hline $\mathrm{CCNF}$ & 5,58 & 5,61 & 5,39 & 4,67 & 5,91 & 0,35 & 0,95 & 0,54 & 0,26 & 0,20 \\
\hline CAMI & 3,72 & 3,91 & 3,98 & 3,72 & 4,05 & 0,09 & 0,14 & 0,02 & 0,23 & 0,06 \\
\hline CNDT & 11,39 & 11,21 & 11,90 & 10,64 & 11,89 & 0,34 & 0,72 & 0,30 & 0,78 & 0,02 \\
\hline \multicolumn{11}{|c|}{ Consumo dos Nutrientes, $\%$ da MS } \\
\hline $\mathrm{CPB}$ & 16,06 & 16,27 & 15,77 & 15,81 & 15,53 & 0,05 & 0,53 & 0,54 & 0,60 & 0,16 \\
\hline CFDN & 38,09 & 37,55 & 38,39 & 37,55 & 38,39 & 0,02 & 0,83 & 0,21 & 0,51 & 0,16 \\
\hline CCNF & 34,34 & 34,31 & 31,59 & 28,51 & 34,64 & 0,35 & 0,95 & 0,54 & 0,26 & 0,20 \\
\hline CAMI & 22,89 & 23,91 & 23,33 & 22,71 & 23,74 & 0,09 & 0,14 & 0,02 & 0,23 & 0,06 \\
\hline CNDT & 70,09 & 68,56 & 69,75 & 64,96 & 69,70 & 0,34 & 0,72 & 0,30 & 0,78 & 0,02 \\
\hline \multicolumn{11}{|c|}{ Digestibilidade aparente $^{5}, \% \mathrm{MS}$} \\
\hline DAMS & 66,57 & 65,25 & 69,51 & 68,46 & 66,46 & 1,42 & 0,52 & 0,95 & 0,19 & 0,61 \\
\hline DAPB & 64,02 & 63,76 & 69,07 & 67,27 & 64,40 & 1,18 & 0,87 & 0,82 & 0,01 & 0,30 \\
\hline DAFDN & 49,90 & 45,74 & 53,75 & 49,29 & 47,24 & 2,53 & 0,26 & 0,47 & 0,61 & 0,23 \\
\hline DACNF & 93,07 & 94,33 & 94,57 & 94,41 & 93,95 & 1,09 & 0,43 & 0,57 & 0,30 & 0,91 \\
\hline DAAMI & 90,74 & 90,96 & 91,21 & 90,69 & 86,96 & 0,54 & 0,78 & 0,82 & 0,75 & 0,50 \\
\hline
\end{tabular}

${ }^{1} \mathrm{CMS}=$ consumo de matéria seca; $\mathrm{CMO}=$ consumo de matéria orgânica; $\mathrm{CPB}=$ consumo de proteína bruta; $\mathrm{CNDT}=$ consumo de nutrientes digestíveis totais; $\mathrm{CFDN}=$ consumo de fibra em detergente neutro; $\mathrm{CCNF}=$ consumo de carboidrato não fibroso e CAMI = consumo de amido. ${ }^{2} \mathrm{VM}$ : Virginiamicina; MO: Monensina; $\mathrm{VM}^{300}+\mathrm{MO}^{300}$ : Virginiamicina $300 \mathrm{mg} / \mathrm{dia} \mathrm{e}$ Monensina $300 \mathrm{mg} / \mathrm{dia}$; $\mathrm{VM}^{300}+\mathrm{MO}^{150}$ : Virginiamicina 300mg/dia e Monensina $150 \mathrm{mg} / \mathrm{dia}$; C: Controle. ${ }^{3}$ Erro padrão da média. ${ }^{4}$ Constrastes: $\mathrm{C} 1, \mathrm{VM} \times \mathrm{MO}$; $\mathrm{C} 2$, VM x $\mathrm{C}$; $\mathrm{C} 3, \mathrm{VM} \times \mathrm{VM}^{300}+\mathrm{MO}^{300}$ e $\mathrm{VM}^{300}+\mathrm{MO}^{150} ; \mathrm{C}^{2}, \mathrm{VM}^{300}+\mathrm{MO}^{300} \times$ $\mathrm{VM}^{300}+\mathrm{MO}^{150} .{ }^{5} \mathrm{DAMS}=$ digestibilidade aparente da matéria seca; $\mathrm{DAPB}=$ digestibilidade aparente da proteína bruta; DAFDN = digestibilidade aparente da fibra em detergente neutro; DACNF = digestibilidade aparente do carboidrato não fibroso e DAAMI = digestibilidade aparente do amido.

O consumo de matéria seca (CMS) foi de 16,25 a 17,06 kg de MS/dia. Não foi observada diferença ( $\mathrm{P}>0,05$; Tabela 2) entre as dietas experimentais. Contudo, no $\mathrm{C} 4$ foi observada uma tendência para o maior CMS do grupo $\mathrm{VM}^{300}+\mathrm{MO}^{300}$, esta observação contribui para explicar o maior consumo de amido deste grupo comentado anteriormente. $\mathrm{O}$ impacto do uso de aditivos sobre o CMS não é bem determinado. Até o momento os estudos mostram haver grande influência da composição e 
características físicas dos componentes da dieta influenciando diretamente o consumo dos animais (Green et al., 1999; Ipharraguerre \& Clark, 2003; Sauer et al., 1989).

Não houve diferença para a digestibilidade aparente da MS, da FDN, do CNF e do Amido. No entanto, os valores da digestibilidade aparente da proteína bruta (DAPB) foram diferentes (P < 0,05; Tabela 2) no $\mathrm{C} 3$ que indica superioridade dos grupos que utilizaram VM e $\mathrm{MO}$ associadas quando comparados ao grupo suplementado apenas com VM. A maior DAPB para a dieta contendo os aditivos associados (VM e MO) pode ser justificada pelo efeito de menor deaminação de proteínas, que acarreta um maior aporte de proteínas ao intestino delgado. Além disto, segundo Borges (2011) a adição de VM em dietas de frangos de corte proporciona maior desenvolvimento das vilosidades e das criptas do duodeno e jejuno ampliando a superfície de digestão e absorção dos nutrientes. Somado a isto em suínos, a virginiamicina melhora a digestão da MS, do N e energia metabolizável e minerais (Vervaeke et al., 1979). Esses benefícios foram atribuídos ao prolongamento do tempo de retenção intestinal e à redução da evolução bacteriana intestinal e produção de ácido orgânico correspondente. Hipotetiza-se que essas alterações também possam ocorrer nas vacas em lactação e com isso resultar na maior digestibilidade aparente da proteína nesses animais.

\section{Desempenho}

Foi observada diferença para a produção de leite $(<0,05$; Tabela 3) nos $\mathrm{C} 2$ e C3 que indicam maior produção de leite no grupo controle e nos grupos suplementados com VM e MO associadas quando comparados a virginiamicina. Estes resultados podem ser explicados de formas diferentes, a superioridade do grupo controle pode ser justificada pelo maior consumo de amido por esses animais, proporcionando maior produção de proteína microbiana e de ácidos graxos voláteis. Já a maior produção de leite observada para os grupos que utilizaram os aditivos associados pode ser explicada pelo efeito sinérgico destes dois aditivos relacionado à modulação da fermentação ruminal, que resultou na maior produção de propionato e de ácidos graxos totais, e das alterações intestinais que resultaram na maior digestibilidade aparente da proteína nesses tratamentos quando comparados a virginiamicina. Erasmus et al. (2008) avaliaram a inclusão de VM e/ou monensina na dieta a base de feno de alfafa fornecido para vacas holandesas e suplementado com $15,50 \mathrm{~kg}$ de MS de concentrado/dia. Da mesma forma, os autores não observaram diferenças para produção de leite em resposta a inclusão da VM (36,6 kg/d) ou da MO (37,4 kg/d) quando adicionadas a dieta e comparadas ao grupo controle $(38,9 \mathrm{~kg} / \mathrm{d})$. Todavia, quando associadas $(\mathrm{VM}+\mathrm{MO})$ e adicionadas à dieta foi observada maior produção de leite $(41,2 \mathrm{~kg} / \mathrm{d})$. Os autores justificam os efeitos positivos da associação dos dois aditivos na estabilização da ingestão alimentar e da fermentação ruminal, juntamente com um potencial efeito pós-ruminal da VM.

Os teores de gordura, de proteína, de lactose e de sólidos totais do leite não diferiram entre os animais sob as diferentes dietas experimentais ( $\mathrm{P}>0,05$; Tabela 3 ). Contudo, no $\mathrm{C} 2$ é possível observar tendência para superioridade do grupo controle quanto as produções em $\mathrm{kg}$ de gordura e proteína e produções de leite corrigidas para os teores de sólidos. Estes resultados podem ser explicados também pela maior produção de leite neste grupo comparado as demais dietas experimentais. Entretanto, estes resultados divergem dos encontrados por Erasmus et al. (2008) que observaram maiores produções de gordura e proteína quando a VM foi utilizada associada a MO.

Os dados de eficiência alimentar evidenciaram diferenças estatísticas nos $\mathrm{C} 2$ e C3, o que demonstra melhor eficiência alimentar para o grupo controle e os grupos que utilizaram os aditivos associados quando comparados com os grupos que receberam apenas VM, o que pode ser explicado pela maior produção de leite e ausência de diferença no CMS encontrada nestes grupos.

A eficiência do uso do nitrogênio para produção de leite, utilizada por alguns autores (Broderick, 2003; Huhtanen \& Hristov, 2009) como ferramenta de avaliação de eficiência em propriedades leiteiras também foi calculada. A mesma se refere à capacidade da vaca em secretar proteína no leite a partir do nitrogênio consumido. No presente estudo não foram observadas diferenças entre os tratamentos $(\mathrm{P}>$ 0,05; Tabela 3). Contudo, o C2 apresentou tendência em maior eficiência da utilização do nitrogênio 
para o grupo controle, o que pode ser explicado pela maior disponibilidade de energia no rúmen destes animais que consumiram maior quantidade amido quando comparado às demais dietas experimentais.

Tabela 3. Desempenho, composição do leite e eficiências alimentar e de utilização de $\mathrm{N}$ nas dietas experimentais com ou sem inclusão de virginiamicina e/ou monensina.

\begin{tabular}{|c|c|c|c|c|c|c|c|c|c|c|}
\hline \multirow{2}{*}{ Itens $^{1}$} & \multicolumn{5}{|c|}{ Dietas $^{2}$} & \multirow{2}{*}{$\mathrm{EPM}^{3}$} & \multicolumn{4}{|c|}{ Contrastes $^{4}($ Valor de P) } \\
\hline & VM & MO & $\mathrm{VM}^{300}+\mathrm{MO}^{300}$ & $\mathrm{VM}^{300}+\mathrm{MO}^{150}$ & $\mathrm{C}$ & & $\mathrm{C} 1$ & $\mathrm{C} 2$ & $\mathrm{C} 3$ & $\mathrm{C} 4$ \\
\hline $\mathrm{CMS}, \mathrm{kg} / \mathrm{d}$ & 16,25 & 16,35 & 17,06 & 16,38 & 17,06 & 0,35 & 0,78 & 0,12 & 0,29 & 0,19 \\
\hline CMS, \% PV & 3,45 & 3,42 & 3,56 & 3,45 & 3,53 & 0,29 & 0,67 & 0,19 & 0,24 & 0,08 \\
\hline $\mathrm{CMO}, \mathrm{kg} / \mathrm{d}$ & 17,20 & 17,08 & 17,42 & 17,42 & 17,51 & 0,19 & 0,65 & 0,27 & 0,36 & 1,00 \\
\hline Leite, $\mathrm{kg} / \mathrm{d}$ & 23,77 & 24,33 & 25,93 & 25,73 & 26,57 & 0,71 & 0,58 & 0,01 & 0,03 & 0,84 \\
\hline $\mathrm{LCE}, \mathrm{kg} / \mathrm{d}$ & 24,54 & 24,96 & 23,91 & 25,91 & 28,49 & 0,92 & 0,85 & 0,10 & 0,85 & 0,38 \\
\hline $\mathrm{LCG} 3,5, \mathrm{~kg} / \mathrm{d}$ & 25,18 & 25,48 & 25,63 & 27,43 & 29,01 & 1,38 & 0,87 & 0,06 & 0,41 & 0,34 \\
\hline Gordura, $\%$ & 3,88 & 3,88 & 3,58 & 4,05 & 4,23 & 0,21 & 0,98 & 0,26 & 0,79 & 0,14 \\
\hline Gordura, kg/d & 0,94 & 0,94 & 0,91 & 1,02 & 1,10 & 0,07 & 0,96 & 0,10 & 0,71 & 0,24 \\
\hline Proteína, \% & 3,32 & 3,33 & 3,00 & 3,23 & 3,47 & 0,05 & 0,95 & 0,42 & 0,24 & 0,24 \\
\hline Proteína, kg/d & 0,79 & 0,81 & 0,77 & 0,83 & 0,91 & 0,05 & 0,78 & 0,08 & 0,86 & 0,40 \\
\hline Lactose, $\%$ & 4,42 & 4,51 & 4,03 & 4,10 & 4,51 & 0,05 & 0,75 & 0,75 & 0,14 & 0,77 \\
\hline Lactose, $\mathrm{kg} / \mathrm{d}$ & 1,05 & 1,10 & 1,05 & 1,06 & 1,20 & 0,07 & 0,63 & 0,14 & 0,98 & 0,90 \\
\hline Sólidos, \% & 8,80 & 8,88 & 7,99 & 8,32 & 9,08 & 0,05 & 0,93 & 0,34 & 0,29 & 0,31 \\
\hline Sólidos, kg/d & 2,09 & 2,16 & 2,06 & 2,13 & 2,40 & 0,06 & 0,88 & 0,58 & 0,15 & 0,51 \\
\hline NUL, mg/dL & 9,8 & 8,8 & 9,2 & 8,3 & 9,1 & 0,09 & 0,55 & 0,86 & 0,28 & 0,25 \\
\hline Leite/CMS & 1,21 & 1,24 & 1,27 & 1,31 & 1,30 & 0,02 & 0,38 & 0,01 & 0,01 & 0,21 \\
\hline N Leite/N consumido & 0,31 & 0,31 & 0,29 & 0,32 & 0,35 & 0,02 & 0,87 & 0,09 & 0,88 & 0,24 \\
\hline
\end{tabular}

${ }^{1} \mathrm{CMS}=$ consumo de matéria seca; $\mathrm{CMO}$ = consumo de matéria orgânica; LCE = leite corrigido para energia; LCG 3,5: leite corrigido para 3,5\% de gordura; $\mathrm{NUL}=$ nitrogênio ureico do leite. ${ }^{2} \mathrm{VM}$ : Virginiamicina; $\mathrm{MO}$ : Monensina; $\mathrm{VM}^{300}+\mathrm{MO}^{300}$ : Virginiamicina 300 $\mathrm{mg} /$ dia e Monensina $300 \mathrm{mg} / \mathrm{dia} ; \mathrm{VM}^{300}+\mathrm{MO}^{150}$ : Virginiamicina $300 \mathrm{mg} /$ dia e Monensina $150 \mathrm{mg} / \mathrm{dia}$; C: Controle. ${ }^{3}$ Erro padrão da média. ${ }^{4}$ Contrastes: $\mathrm{C} 1, \mathrm{VM}$ x MO; $\mathrm{C} 2, \mathrm{VM}$ x C; C3, VM x VM ${ }^{300}+\mathrm{MO}^{300} \mathrm{e} \mathrm{VM}^{300}+\mathrm{MO}^{150}$; 4 , $\mathrm{VM}^{300}+\mathrm{MO}^{300} \mathrm{x} \mathrm{VM}^{300}+\mathrm{MO}^{150}$.

Os valores de nitrogênio ureico no plasma (NUP) são apresentados na Figura 1. Os resultados evidenciam maiores teores de NUP nos animais que consumiam a dieta $\mathrm{VM}^{300}+\mathrm{MO}^{300} \mathrm{e} \mathrm{VM}^{300}+\mathrm{MO}^{150}$ durante todo o período de coleta. Estes valores coincidem com o perfil apresentado pelos resultados de $\mathrm{N}$ amoniacal que também se justificam pelos efeitos do uso desses aditivos. Os valores se mantiveram acima de 13,19 mg/dL próximos aos valores médios descritos por Broderick \& Clayton (1997) para vacas com média de $25 \mathrm{~kg}$ de leite por dia. No presente estudo mesmo os valores mais baixos $(13,19$ $\mathrm{mg} / \mathrm{dL}$ ) se apresentam dentro da faixa relatada na literatura para vacas produzindo em média $25 \mathrm{~kg}$ de leite por dia.

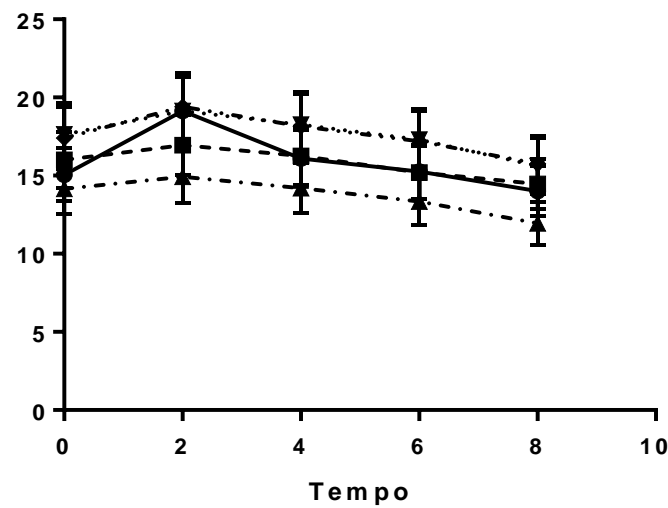

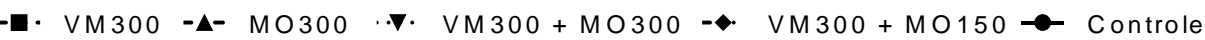

Figura 1. Nitrogênio ureico plasmático $(\mathrm{mg} / \mathrm{dL})$ de vacas leiteiras alimentadas com dietas experimentais com ou sem a inclusão de virginiamicina e/ou monensina em função do tempo referente à primeira alimentação. 


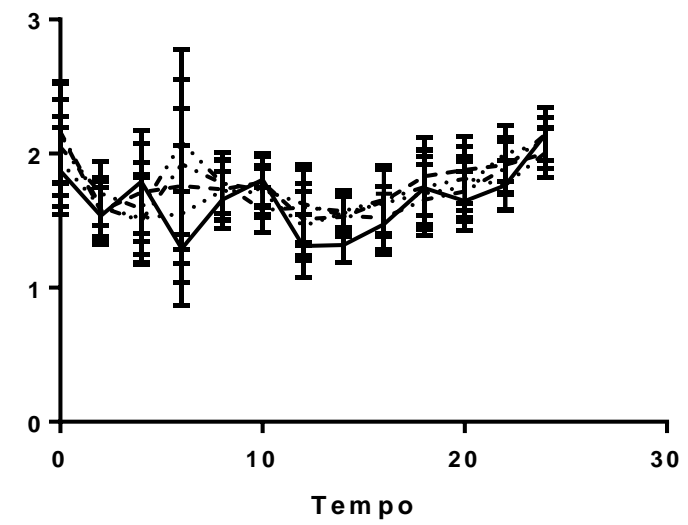

-.VVM300 -. MO300 $\cdots$ VM VM00

Figura 2. Razão acetato/propionato de vacas leiteiras alimentadas com dietas experimentais com ou sem a inclusão de virginiamicina e/ou monensina em função do tempo referente à primeira alimentação.

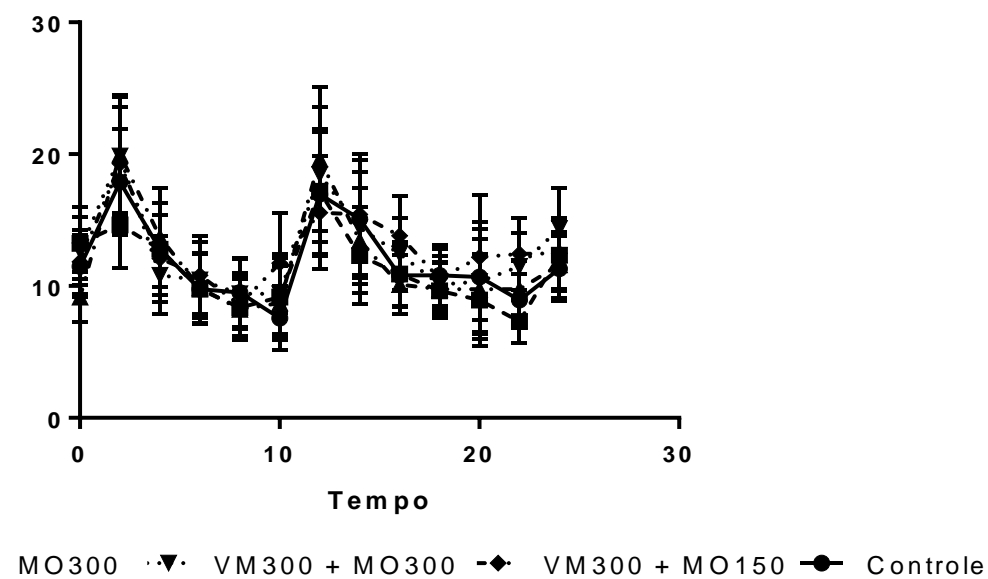

Figura 3. Nitrogênio amoniacal $(\mathrm{mg} / \mathrm{dL})$ de vacas leiteiras alimentadas com dietas experimentais com ou sem a inclusão de virginiamicina e/ou monensina em função do tempo referente à primeira alimentação.

\section{Parâmetros ruminais}

Foram observados valores médios de $\mathrm{pH}$ no rúmen próximos de 6,1 para todas as dietas experimentais (Tabela 4), o que indica ausência de acidose subclínica mesmo com uma proporção baixa de volumoso em relação ao concentrado na MS (35:65). Estes resultados podem ter ocorrido em decorrência do volumoso utilizado, o tamanho de partícula (fibra fisicamente efetiva), a fibra efetiva dos alimentos utilizados como o caroço de algodão e também o fracionamento no fornecimento da dieta. O C4 evidenciou a superioridade dos animais suplementados com a VM e a MO associadas nas doses de 300 e $150 \mathrm{mg} / \mathrm{vaca} /$ dia, respectivamente, em manter os valores de $\mathrm{pH}$ ruminal mais elevados quando comparado a associação na dose de $300 \mathrm{mg} / \mathrm{vaca} / \mathrm{dia}$. A dose mais elevada de monensina de MO pode ser tóxica aos microrganismos ruminais responsáveis pela manutenção dos valores de $\mathrm{pH}$ mais elevados.

Foi observado comportamento semelhante para os valores de $\mathrm{pH}$ fecal. $\mathrm{O}$ maior valor encontrado para os animais alimentados com a dieta que utilizou a associação $\mathrm{VM}^{300}+\mathrm{MO}^{150}$ evidenciado pela diferença no $\mathrm{C} 4(\mathrm{P}<0,05)$, seguido pelo indicativo de tendência do $\mathrm{C} 1$ contraste que evidência maior $\mathrm{pH}$ fecal do grupo VM quando comparado ao grupo MO. Segundo Channon et al. (2004) em dietas com alta inclusão de concentrado o $\mathrm{pH}$ fecal pode ser um indicador para avaliar a eficiência em digestibilidade e absorção de carboidratos, principalmente amido, no trato digestivo de ruminantes. $\mathrm{Da}$ mesma forma, Montano et al. (2015) avaliando parâmetros ruminais em novilhos com dieta de feno de alfafa e inclusão de alto teor de concentrado processado e adição de VM (26 mg/kg de MS) e MO (34 
$\mathrm{mg} / \mathrm{kg}$ de MS) observaram valores de $\mathrm{pH}$ ruminal superiores para o grupo suplementado com os estes dois aditivos associados.

No $\mathrm{C} 2$ foi observado maior produção de propionato, butirato e AGV totais no grupo controle quando comparado ao grupo VM, o que pode ser explicado pelo maior consumo de amido deste grupo. $\mathrm{O}$ aumento de propionato era esperado nos grupos com a utilização de aditivos pela seleção das bactérias produtoras deste ácido (Coe et al., 1999); no entanto, este efeito não foi observado.

Tabela 4. Parâmetros ruminais e pH fecal de vacas leiteiras nas dietas experimentais com ou sem inclusão de virginiamicina e/ou monensina

\begin{tabular}{|c|c|c|c|c|c|c|c|c|c|c|c|}
\hline \multirow{2}{*}{ Itens } & \multicolumn{5}{|c|}{ Dietas $^{1}$} & \multirow{2}{*}{$\mathrm{EPM}^{2}$} & \multicolumn{5}{|c|}{ Contrastes $^{4}$} \\
\hline & $\mathrm{VM}$ & $\mathrm{MO}$ & $\mathrm{VM}^{300}+\mathrm{MO}^{300}$ & $\mathrm{VM}^{300}+\mathrm{MO}^{150}$ & $\mathrm{C}$ & & $\mathrm{T} \times \mathrm{H}^{3}$ & $\mathrm{C} 1$ & $\mathrm{C} 2$ & $\mathrm{C} 3$ & $\mathrm{C} 4$ \\
\hline$\overline{\mathrm{pH} \text { ruminal }}$ & 6,19 & 6,11 & 6,16 & 6,29 & 6,18 & 0,01 & 0,81 & 0,17 & 0,93 & 0,45 & 0,02 \\
\hline $\mathrm{pH}$ fecal & 6,19 & 6,34 & 6,12 & 6,35 & 6,11 & 0,06 & - & 0,08 & 0,33 & 0,52 & 0,01 \\
\hline $\begin{array}{l}\mathrm{N}-\mathrm{NH}_{3} \\
(\mathrm{mg} / \mathrm{dL})\end{array}$ & 11,08 & 11,65 & 12,4 & 12,64 & 11,64 & 0,06 & 0,01 & 0,26 & 0,25 & 0,01 & 0,79 \\
\hline Acetato $(\mathrm{mMol} / \mathrm{dL})$ & 6,17 & 6,37 & 6,26 & 6,59 & 6,70 & 0,08 & 0,17 & 0,56 & 0,13 & 0,40 & 0,34 \\
\hline $\begin{array}{l}\text { Propionato } \\
(\mathrm{mMol} / \mathrm{dL})\end{array}$ & 3,57 & 3,71 & 3,71 & 3,99 & 4,22 & 0,08 & 0,47 & 0,56 & 0,01 & 0,16 & 0,21 \\
\hline Butirato $(\mathrm{mMol} / \mathrm{dL})$ & 0,69 & 0,70 & 0,76 & 0,74 & 0,80 & 0,07 & 0,10 & 0,86 & 0,01 & 0,13 & 0,60 \\
\hline Acetato/Propionato & 1,72 & 1,75 & 1,68 & 1,65 & 1,58 & 0,07 & 0,01 & 0,96 & 0,24 & 0,44 & 0,62 \\
\hline $\mathrm{AGV}(\mathrm{mMol} / \mathrm{dL})$ & 10,44 & 10,78 & 10,73 & 11,31 & 11,72 & 0,06 & 0,38 & 0,55 & 0,02 & 0,24 & 0,31 \\
\hline
\end{tabular}

${ }^{1} \mathrm{VM}$ : Virginiamicina; MO: Monensina; $\mathrm{VM}^{300}+\mathrm{MO}^{300}$ : Virginiamicina 300mg/dia e Monensina $300 \mathrm{mg} /$ dia; $\mathrm{VM}^{300}+\mathrm{MO}^{150}$ : Virginiamicina 300mg/dia e Monensina $150 \mathrm{mg} / \mathrm{dia}$; C: Controle. ${ }^{2}$ Erro padrão da média. ${ }^{3}$ Interação tratamento x tempo. ${ }^{4}$ Contrastes: $\mathrm{C} 1, \mathrm{VM}$ x MO; $\mathrm{C} 2, \mathrm{VM}$ x C; C3, VM x VM ${ }^{300}+\mathrm{MO}^{300} \mathrm{e} \mathrm{VM}^{300}+\mathrm{MO}^{150}$; $44, \mathrm{VM}^{300}+\mathrm{MO}^{300}$ x VM $\mathrm{VM}^{300}+\mathrm{MO}^{150}$.

Para os valores de nitrogênio amoniacal no $\mathrm{C} 3$ foi observada maior concentração para os grupos que utilizaram VM e MO associados (P > 0,05; Tabela 4) quando comparado ao grupo recebeu apenas VM. Não era esperado o aumento nos teores de $\mathrm{N}$ amoniacal nos grupos que receberam os dois aditivos uma vez que esses aditivos têm conhecida capacidade de inibir o processo de deaminação e com isso da produção ruminal de amônia (Montano et al., 2015; Mullins et al., 2012; Schelling, 1984).

\section{Conclusão}

A adição de virginiamicina não influenciou o desempenho de vacas leiteiras em dietas com alta inclusão de concentrado. Contudo a virginiamicina associada à monensina demonstrou efeito aditivo por ser a dieta experimental que mais influenciou positivamente os efeitos sobre a fermentação e a saúde ruminal, a eficiência alimentar, a digestibilidade da proteína bruta e a produção de leite. Mais estudos são necessários para definição da melhor proporção entre as doses desta associação.

\section{Referências bibliográficas}

AOAC. (2005). - Association Official Analytical Chemist (2005) (Official Methods of Analysis (18th ed.) ed.). Gaitherburg, Maryland, USA: AOAC.

Borges, L. L. (2011). Efeito de dietas contendo virginiamicina sobre o desempenho, o rendimento de carcaça e a morfometria intestinal de frangos de corte. Master of Science, Universidade Estadual Paulista, Jaboticabal, São Paulo, Brasil.

Broderick, G. A. (2003). Effects of varying dietary protein and energy levels on the production of lactating dairy cows. Journal of Dairy Science, 86(4):1370-1381.

Broderick, G. A. \& Clayton, M. K. (1997). A statistical evaluation of animal and nutritional factors influencing concentrations of milk urea nitrogen. Journal of Dairy Science, 80(11):2964-2971.

Callaway, T. R., Edrington, T. S., Rychlik, J. L., Genovese, K. J., Poole, T. L., Jung, Y. S., . . Nisbet, D. J. (2003). Ionophores: their use as ruminant growth promotants and impact on food safety. Current Issues in Intestinal Microbiology, 4(2):43-51. 
Channon, A. F., Rowe, J. B. \& Herd, R. M. (2004). Genetic variation in starch digestion in feedlot cattle and its association with residual feed intake. Australian Journal of Experimental Agriculture, 44(5):469-474.

Coe, M. L., Nagaraja, T. G., Sun, Y. D., Wallace, N., Towne, E. G., Kemp, K. E. \& Hutcheson, J. P. (1999). Effect of virginiamycin on ruminal fermentation in cattle during adaptation to a high concentrate diet and during an induced acidosis. Journal of Animal Science, 77(8):2259-2268.

DePeters, E. J. \& Ferguson, J. D. (1992). Nonprotein nitrogen and protein distribution in the milk of cows. Journal of Dairy Science, 75(11):3192-3209.

Detmann, E., Souza, M., Valadares Filho, S., Queiroz, A., Berchielli, T., Saliba, E., . . Azevedo, J. (2012). Métodos para análise de alimentos. Minas Gerais: Suprema.

Duffield, T. F., Merrill, J. K. \& Bagg, R. N. (2012). Meta-analysis of the effects of monensin in beef cattle on feed efficiency, body weight gain, and dry matter intake. Journal of Animal Science, 90(12):4583-4592.

Erasmus, L. J., Muya, C., Erasmus, S., Coertze, R. F. \& Catton, D. G. (2008). Effect of virginiamycin and monensin supplementation on performance of multiparous Holstein cows. Livestock Science, 119(1-3):107-115.

González, L. A., Manteca, X., Calsamiglia, S., Schwartzkopf-Genswein, K. S. \& Ferret, A. (2012). Ruminal acidosis in feedlot cattle: Interplay between feed ingredients, rumen function and feeding behavior (a review). Animal Feed Science and Technology, 172(1-2):66-79. doi: http://dx.doi.org/10.1016/j.anifeedsci.2011.12.009

Green, B. L., McBride, B. W., Sandals, D., Leslie, K. E., Bagg, R. \& Dick, P. (1999). The impact of a monensin controlled-release capsule on subclinical ketosis in the transition dairy cow. Journal of Dairy Science, 82(2):333-342.

Hall, M. B., Hoover, W. H., Jennings, J. P. \& Webster, T. K. M. (1999). A method for partitioning neutral detergent soluble carbohydrates. Journal of the Science of Food and Agriculture, 70(15):2079-2086. doi: https://doi.org/10.1002/(SICI)1097-0010(199912)79:15<2079::AIDJSFA502>3.0.CO;2-Z.

Huhtanen, P. \& Hristov, A. N. (2009). A meta-analysis of the effects of dietary protein concentration and degradability on milk protein yield and milk $\mathrm{N}$ efficiency in dairy cows. Journal of Dairy Science, 92(7):3222-3232.

Ipharraguerre, I. R. \& Clark, J. H. (2003). Usefulness of ionophores for lactating dairy cows: a review. Animal Feed Science and Technology, 106(1-4):39-57. doi: 10.1016/S0377-8401(03)00065-8

McGuffey, R. K., Richardson, L. F. \& Wilkinson, J. I. D. (2001). Ionophores for dairy cattle: current status and future outlook. Journal of Dairy Science, 84E194-E203.

Montano, M. F., Manriquez, O. M., Salinas-Chavira, J., Torrentera, N. \& Zinn, R. A. (2015). Effects of monensin and virginiamycin supplementation in finishing diets with distiller dried grains plus solubles on growth performance and digestive function of steers. Journal of Applied Animal Research, 43(4):417-425. doi: http://dx.doi.org/10.1080/09712119.2014.978785.

Mullins, C. R., Mamedova, L. K., Brouk, M. J., Moore, C. E., Green, H. B., Perfield, K. L., . . Bradford, B. J. (2012). Effects of monensin on metabolic parameters, feeding behavior, and productivity of transition dairy cows. Journal of Dairy Science, 95(3):1323-1336. doi: http://dx.doi.org/10.3168/jds.2011-4744

Nagaraja, T. G., Newbold, C. J., Van Nevel, C. J. \& Demeyer, D. I. (1997). Manipulation of ruminal fermentation The rumen microbial ecosystem (pp. 523-632): Springer.

Nagaraja, T. G. \& Taylor, M. B. (1987). Susceptibility and resistance of ruminal bacteria to antimicrobial feed additives. Applied and Environmental Microbiology, 53(7):1620-1625.

NRC. (2001). Nutrient Requirements of Dairy Cattle (7th rev. ed.). Washington: Natl. Acad. Press, Washington, DC.

SAS. (2004). SAS/STAT User guide, Version 9.1.2. Cary, NC, USA: SAS Institute Inc.

Sauer, F. D., Kramer, J. K. G. \& Cantwell, W. J. (1989). Antiketogenic effects of monensin in early lactation. Journal of Dairy Science, 72(2):436-442. 
Schelling, G. T. (1984). Monensin mode of action in the rumen. Journal of Animal Science, 58(6):1518-1527.

Van Soest, P. J., Robertson, J. B. \& Lewis, B. A. (1991). Methods for dietary fiber, neutral detergent fiber, and nonstarch polysaccharides in relation to animal nutrition. Journal of Dairy Science, 74(10):3583-3597. doi: http://dx.doi.org/10.3168/jds.S0022-0302(91)78551-2.

Vervaeke, I. J., Decuypere, J. A., Dierick, N. A. \& Henderickx, H. K. (1979). Quantitative in vitro evaluation of the energy metabolism influenced by virginiamycin and spiramycin used as growth promoters in pig nutrition. Journal of Animal Science, 49(3):846-856.

Wu, S. H. W. \& Papas, A. (1997). Rumen-stable delivery systems. Advanced Drug Delivery Reviews, 28(3):323-334. doi: http://dx.doi.org/10.1016/S0169-409X(97)00087-2.

Recebido: 25 de junho, 2019.

Aprovado: 27 de julho, 2019.

Publicado: 3 de janeiro, 2020.

Licenciamento: Este artigo é publicado na modalidade Acesso Aberto sob a licença Creative Commons Atribuição 4.0 (CC-BY 4.0), a qual permite uso irrestrito, distribuição, reprodução em qualquer meio, desde que o autor e a fonte sejam devidamente creditado. 